\title{
Ketamine Use allows Noninvasive Ventilation in Distressed Patients with Acute Decompensated Heart Failure
}

\author{
Ankur Verma ${ }^{1}$, Abhishek Snehy $^{2}$, Amit Vishen $^{3}$, Wasil Rasool Sheikh ${ }^{4}$, Meghna Haldar ${ }^{5}$, Sanjay Jaiswal ${ }^{6}$
}

\begin{abstract}
Acute decompensated heart failure (ADHF) is responsible for a heavy clinical load on busy emergency departments (EDs) across the globe and especially in India. ADHF patients may present with severe respiratory distress, dyspnea, hypoxia, and high and low blood pressures. Managing the airway of such patients can at times be challenging. Nasal cannulae, face mask, and noninvasive positive pressure ventilation (NIPPV) are the cornerstones of providing oxygenation and ventilation to such patients while some extreme cases may require endotracheal intubation and mechanical ventilation.

An elderly female in severe respiratory distress and altered sensorium presented to our ED and had to be administered ketamine to facilitate proper NIPPV and avoid mechanical ventilation. She was weaned off the NIPPV in the ED itself over the next four hours. There are some case reports of using ketamine for NIPPV in asthma exacerbations, but none for the use in ADHF. Avoiding invasive mechanical ventilation via endotracheal intubation should be a constant goal and the last resort.

Keywords: Dissociation, Heart failure, Ketamine, NIPPV

Indian Journal of Critical Care Medicine (2019): 10.5005/jp-journals-10071-23153
\end{abstract}

\section{INTRODUCTION}

Any acute or insidious progression of heart failure (HF) requiring urgent medical attention is what defines ADHF. ${ }^{1}$ It is responsible for a heavy clinical load and increases the burden on healthcare and EDs across the globe. Even after the availability of evidencebased medical therapies using nitroglycerine, loop diuretics, levosimendan, etc., the in-hospital mortality across the United States and Europe is $4-7 \% .^{2-4}$ The acute failure registry (AFAR) study conducted in India showed a $38 \%$ mortality of ADHF patients admitted. ${ }^{1}$ Dyspnea is usually the most common symptom with which patients of ADHF present to the ED. Patients can present in altered mental status (AMS) due to hypoxia, which can make standard therapies like noninvasive ventilation and their outcomes difficult to achieve. Patients with ADHF presenting in respiratory distress will require stabilization of their airway and breathing. Oxygen supplementation, especially through NIPPV, has been shown to reduce the need for intubation. ${ }^{5}$ We describe a case where ketamine was used to allow compliance with NIPPV in a severely distressed patient of ADHF.

\section{Case History}

A 71-year-old female presented to our ED in severe respiratory distress and AMS. She was a known diabetic, hypertensive, post permanent pacemaker implantation, and was on diuretics and angiotensin-converting enzyme (ACE) inhibitors for dilated cardiomyopathy with a baseline ejection fraction (EF) of $25 \%$. On arrival she was tachypneic with respiratory rate of 40 breaths/ minute, heart rate of 120 beats/minute, blood pressure $200 / 110 \mathrm{~mm}$ $\mathrm{Hg}$, and oxygen saturation as indicated by pulse oximeter was $75 \%$ on room air. Her temperature was $98^{\circ} \mathrm{F}$ and random blood sugar was $156 \mathrm{mg} / \mathrm{dL}$. Systemic examination revealed reduced air entry in both lungs and coarse crepitations bilaterally. Oxygen supplementation was started at $8 \mathrm{~L} /$ minute via reservoir mask. Intravenous

\footnotetext{
1-6Department of Emergency Medicine, Max Super Specialty Hospital, Patparganj, New Delhi, India
}

Corresponding Author: Ankur Verma, Department of Emergency Medicine, Max Super Specialty Hospital, Patparganj, New Delhi, India, e-mail:anksv25@gmail.com

How to cite this article: Verma A, Snehy $A$, et al. Ketamine Use Allows Noninvasive Ventilation in Distressed Patients with Acute Decompensated Heart Failure. Indian J Crit Care Med 2019;23(4): 191-192.

Source of support: Nil

Conflict of interest: None

furosemide $60 \mathrm{mg}$ and nitroglycerine infusion ( $20 \mu \mathrm{g} /$ minute) were given. She did not respond to oxygen supplementation and decision to start her on bilevel positive airway pressure (BiPAP) ventilation was made. Consent for intubation was also taken from the family. Attempts to start BiPAP failed as the patient would push away the mask and was increasingly agitated. Intravenous bolus dose of $30 \mathrm{mg}$ ketamine, which lightly sedated the patient, was given and BiPAP was started. After about 30 minutes the patient awoke and was given another bolus of $15 \mathrm{mg}$ of ketamine while the patient was still on BiPAP. One hour later, patient was conscious and oriented and showed good tolerance to BiPAP. Her respiratory rate settled to 16 breaths/minute with saturation of $95 \%$ on BiPAP, heart rate of 78 beats/minute, and blood pressure of 130/70 mm $\mathrm{Hg}$. Four hours after arrival to the ED she was weaned off the BiPAP and nitroglycerine and maintained oxygen saturation of $100 \%$ on 2 $\mathrm{L} /$ minute via nasal cannula, respiration of 16 breaths/minute, heart rate 68 beats/minute, and blood pressure of $140 / 80 \mathrm{~mm} \mathrm{Hg}$. The initial electrocardiogram (ECG) had shown a paced rhythm with no acute changes. Two-dimensional echocardiography done in the $\mathrm{ED}$ revealed global hypokinesia with $\mathrm{EF}$ of $25 \%$. Blood gas at the time of arrival revealed severe type 1 respiratory failure. Laboratory tests were significant for $\mathrm{N}$-terminal pro-brain natriuretic peptide

(c) The Author(s). 2019 Open Access This article is distributed under the terms of the Creative Commons Attribution 4.0 International License (http://creativecommons. org/licenses/by/4.0/), which permits unrestricted use, distribution, and non-commercial reproduction in any medium, provided you give appropriate credit to the original author(s) and the source, provide a link to the Creative Commons license, and indicate if changes were made. The Creative Commons Public Domain Dedication waiver (http://creativecommons.org/publicdomain/zero/1.0/) applies to the data made available in this article, unless otherwise stated. 
(NT-ProBNP) of $13,900 \mathrm{pg} / \mathrm{mL}$. She was shifted to the cardiac unit for further management. The patient was managed conservatively and was discharged in a stable condition after 3 days on medications and follow-up advice.

\section{Discussion}

Ketamine and NIPPV have been used in asthma exacerbations as a temporizing measure to avoid mechanical ventilation. ${ }^{6}$ Although not many studies have been done, there are reports ${ }^{6,7}$ where the use of ketamine has facilitated the use of NIPPV and the need for endotracheal intubation has been avoided in such patients. It has also been reported that patients presenting to the ED in respiratory distress when induced with ketamine for intubation have responded to oxygenation with NIPPV and did not require endotracheal intubation. ${ }^{7}$ There have been no case reports or studies of having used ketamine in $\mathrm{HF}$ patients to allow compliance with NIPPV. Although there is a level B evidence, as described by the American Heart Association Scientific statement ${ }^{8}$ that ketamine can precipitate HF due to its negative inotropic effects, the evidence remains inconclusive. There have been theoretical concerns that use of NIPPV in patients with AMS increases the risk of aspiration. ${ }^{9}$ however, ketamine-induced sedation leads to the retention of airway reflexes and spontaneous breathing. ${ }^{10}$ Agitated and restless patients presenting with severe respiratory distress can pose a challenge to manage. If a patient does not comply with NIPPV, unwanted endotracheal intubation might be required in the ED.

We believe that ketamine induced dissociative state in the patient with ADHF facilitated NIPPV management in an otherwise uncooperative patient and allowed NIPPV to take effect. This led to the avoidance of an impending intubation. The patient had an uneventful stay in the hospital which could be attributed to the acute management in the ED. As emergency physicians, it should be our goal to avoid intubating patients and putting them on mechanical ventilation when other options may be attempted. Large series and trials would be required to establish the use of ketamine induced sedation in agitated, uncooperative patients of HF to allow compliance with NIPPV. Till then we suggest it to be used with caution in this subset of patients.

\section{References}

1. Seth S, Khanal S, et al. Epidemiology of acute decompensated heart failure in India: The AFAR Study (Acute failure study registry). J Pract Cardiovasc Sci 2015;1:35-38.

2. Gheorghiade $M$, Zannad $F$, et al. Acute heart failure syndromes: current state and framework for future research. Circulation 2005;112(25):3958-3968.

3. Fonarow GC, Abraham WT, et al. Influence of a performanceimprovement initiative on quality of care for patients hospitalized with heart failure: results of the Organized Program to Initiate Lifesaving Treatment in Hospitalized Patients With Heart Failure (OPTIMIZE-HF). Arch Intern Med 2007;167(14):1493-1502.

4. Fonarow GC, Abraham WT, et al. Association between performance measures and clinical outcomes for patients hospitalized with heart failure. JAMA 2007;297(1):61-70.

5. Vital FM, Ladeira MT, et al. Non-invasive positive pressure ventilation (CPAP or bilevel NPPV) for cardiogenic pulmonary oedema. Cochrane Database Syst Rev 2008;(5):CD005351.

6. Shlamovitz GZ, Hawthorne T. Intravenous ketamine in a dissociating dose as a temporizing measure to avoid mechanical ventilation in adult patient with severe asthma exacerbation. J Emerg Med 2011;41(5):492-494.

7. Weingart SD, Trueger $S$, et al. Delayed sequence intubation: a prospective observational study. Ann Emerg Med 2015;65(4):349-355.

8. Page RL, O'Bryant $\mathrm{CL}$, et al. Drugs That May Cause or Exacerbate Heart Failure: A Scientific Statement From the American Heart Association. Circulation 2016;134(6): e32-69.

9. Mani R. Noninvasive ventilation for hypercapnic respiratory failure in COPD: encephalopathy and initial post-support deterioration of $\mathrm{pH}$ and $\mathrm{PaCO}_{2}$ may not predict failure. Indian J Crit Care Med 2005;9:217224.

10. Green SM, Roback MG, et al. Clinical practice guideline for emergency department ketamine dissociative sedation: 2011 update. Ann Emerg Med 2011;57(5):449-461. 